\title{
BERNSTEIN SETS WITH ALGEBRAIC PROPERTIES
}

\author{
MARCIN KYSIAK
}

\begin{abstract}
We construct Bernstein sets in $\mathbb{R}$ having some additional algebraic properties. In particular, solving a problem of Kraszewski, Rałowski, Szczepaniak and Żeberski, we construct a Bernstein set which is a $<\mathfrak{c}$-covering and improve some other results of Rałowski, Szczepaniak and Żeberski on nonmeasurable sets.
\end{abstract}

\section{INTRODUCTION}

A set $B \subseteq \mathbb{R}$ is Bernstein, if it intersects all perfect sets in $\mathbb{R}$, but does not contain any of them. A Bernstein set is a classical example of a nonmeasurable set; a Bernstein set cannot be Lebegsue measurable and cannot have the Baire property. Moreover, if $\mathcal{A}$ is an algebra of subsets of $\mathbb{R}$, having the property that every set which is in $\mathcal{A}$ but not hereditary in $\mathcal{A}$, contains a perfect set, then no Bernstein set can be a member of $\mathcal{A}$. Most natural algebras of subsets of $\mathbb{R}$ have this property (see [5]), therefore Bernstein sets are in a sense universal examples of nonmeasurable sets.

In our paper, we introduce a general method of constructing Bernstein sets having additional algebraic properties. In the following section, we will construct Bernstein sets being $\kappa$-coverings and consider the possibility of constructing partitions of $\mathbb{R}$ into such sets. Our results improve and complement results of [4], as well as answer an open question posed there.

Later, we improve results from [8], where nonmeasurable (in the sense of Lebesgue measurability and Baire property) sets with certain algebraic properties were constructed - we construct Bernstein sets with the same properties.

Our notation is standard. The family of subsets of a set $X$ of cardinality $\kappa$ is denoted by $[X]^{\kappa}$. All groups considered are abelian (we shall not explicitly formulate that assumption), so we stick to the additive notation. If $A, B$ are subsets of a group $G, A+B=\{a+b: a \in A, b \in B\}$ (this is called a complex sum of $A$ and $B$ ) and $a+B=\{a\}+B$, for $a \in G$. Similarly $A-B=\{a-b: a \in A, b \in B\}$. If $H$ is a subgroup of a group $G$, then the quotient group is the family

$$
G / H=\{x+H: x \in G\} \subseteq \mathcal{P}(G),
$$

equipped with the operation of the complex sum. The elements of $G / H$ are called cosets of $H$ and the mapping $\pi: G \rightarrow G / H$, defined by $\pi(x)=x+H$ is called the quotient epimorphism.

Our results are formulated in $\mathbb{R}$, but it is easy too see that they also remain valid in all Polish groups having the structure of a linear space over a countable field, in

2000 Mathematics Subject Classification. 28A05, 54H05, 20K99.

Key words and phrases. Bernstein set, $\kappa$-covering, nonmeasurable set. 
particular in Euclidean spaces $\mathbb{R}^{n}$, all separable Banach spaces and in the Cantor space.

\section{BERNSTEIN SETS AND $\kappa$-COVERINGS}

We begin with the following definition.

Definition 2.1. (Carlson, [1]) A subset $C$ of a group $G$ is a $\kappa$-covering, if for every $X \in[G]^{\kappa}$, there exist $t \in G$ such that $t+X \subseteq C$. A set $X$ is a $<\kappa$-covering, if it is a $\lambda$-covering for every $\lambda<\kappa$.

Coverings have been studied extensively in the case of $\kappa=\omega$ (see [6] and [7]). The following proposition is folklore, but we include it for completeness.

Proposition 2.2. A subset $C$ of a group $G$ is a $\kappa$-covering if and only if $\kappa$ many translates of $G \backslash C$ do not cover $G$.

Proof. Observe that for every two sets $A, B \subseteq G$, we have

$$
A-B=\{t \in G: A \cap(t+B) \neq \emptyset\} .
$$

Therefore $(G \backslash C)-X=G$, for $X \in[G]^{<\kappa}$, if and only if, for no $t \in G$ we have $t+X \subseteq C$.

Our work on $\kappa$-coverings was inspired largely by the following theorem from [4].

Theorem 2.3 (Kraszewski-Rałowski-Szczepaniak-Żeberski). There exists a partition of $\mathbb{R}$ into $\mathfrak{c}$ many $<\mathrm{cf}(\mathfrak{c})$-coverings which are Bernstein sets.

The authors formulated also the following

Question 2.4 (Kraszewski-Rałowski-Szczepaniak-Żeberski). Assume that $\mathfrak{c}>$ $\operatorname{cf}(\mathfrak{c})=\omega_{1}$. Does there exists a Bernstein set which is an $\omega_{1}$-covering?

The main goal of this section is to give a positive answer to this question. We shall also discuss other variants and improvements of Theorem 2.3.

2.1. Coverings in abstract groups. Before we begin constructing Bernstein sets being $\kappa$-coverings, we show two facts on coverings in arbitrary groups. The first one will be useful later in one of our constructions. The analogous method to our approach was used in [4], but the fact was not formulated in such a general setting.

Lemma 2.5. Let $G$ be a group and assume that $\kappa=|G|$ is regular. Then there exists a partition of $G$ into $\kappa$ many $<\kappa$-coverings.

Proof. Write $\kappa$ as a disjoint union of sets $A_{\alpha}$, for $\alpha<\kappa$, with each of $A_{\alpha}$ 's of cardinality $\kappa$. Observe also that, by regularity of $\kappa$, there exists an increasing family $\left\{X_{\alpha}: \alpha<\kappa\right\} \subseteq[G]^{<\kappa}$ such that for every $X \in[G]^{<\kappa}$ there exists $\alpha<\kappa$ such that $X \subseteq X_{\alpha}$.

We construct a partition $\left\{C_{\alpha}: \alpha<\kappa\right\}$ by transfinite induction. In step $\beta<\kappa$, find $t_{\beta} \in G$ in such a way that $t_{\beta}+X_{\beta}$ is disjoint with $\bigcup_{\xi<\beta}\left(t_{\xi}+X_{\xi}\right)$. Observe that this is possible, because for cardinality reason, $\left(\bigcup_{\xi<\beta}\left(t_{\xi}+X_{\xi}\right)\right)-X_{\beta} \neq G$ (see the argument in the proof of Proposition 2.2).

Let $C_{\alpha}=\bigcup_{\beta \in A_{\alpha}}\left(t_{\beta}+X_{\beta}\right)$, for $\alpha<\kappa$. By our construction, these sets are pairwise disjoint. Also, each $C_{\alpha}$ contains copies of cofinally many $X_{\beta}$ 's, so it is a $<\kappa$-covering. We have not guaranteed that $G=\bigcup_{\alpha<\kappa} C_{\alpha}$, but if it is not true, me may add the complement of $\bigcup_{\alpha<\kappa} C_{\alpha}$ to $C_{0}$. 
It turns out, however, that the assumption of regularity of the cardinality of the group is necessary.

Proposition 2.6. If a group $G$ of cardinality $\kappa$ contains two disjoint $<\kappa$-coverings, then $\kappa$ is regular.

Proof. Suppose that $A, B$ are two disjoint $<\kappa$-coverings in $G$, where $|G|=\kappa$. Without loss of generality we can assume that $A \cup B=G$. Observe that, by Proposition 2.2, fewer than $\kappa$ many translates of $A=G \backslash B$ do not cover $G$. Let $G=\bigcup_{\xi<\operatorname{cf}(\kappa)} X_{\xi}$, where $\left|X_{\xi}\right|<\kappa$. For every $\xi<\operatorname{cf}(\kappa)$ we may find $t_{\xi}$ such that $t_{\xi}+A \supseteq X_{\xi}$, therefore we have $G=\bigcup_{\xi<\mathrm{cf}(\kappa)}\left(t_{\xi}+A\right)$. As it was impossible to cover $G$ with fewer than $\kappa$ translates of $A$, we must have $\operatorname{cf}(\kappa)=\kappa$.

2.2. Coverings and Bernstein sets. The main trick in our approach to constructing coverings in $\mathbb{R}$ is the application of the following Proposition. However, its proof is so easy and straightforward that we leave ot to the reader.

Proposition 2.7. Let $G$ be a subgroup of $\mathbb{R}$ and let $\pi: \mathbb{R} \rightarrow \mathbb{R} / G$ be the quotient epimorphism $\pi: x \mapsto x+G$. Then the following conditions are equivalent for $C \subseteq \mathbb{R} / G$ and $\kappa \leq|\mathbb{R} / G|$

(1) $C$ is a $\kappa$-covering in $\mathbb{R} / G$,

(2) $\pi^{-1}[C]$ is a $\kappa$-covering in $\mathbb{R}$.

The following lemma is folklore (see similar arguments in [2]) but we sketch the proof for completeness.

Lemma 2.8. For every cardinal $\kappa$ such that $\omega \leq \kappa \leq \mathfrak{c}$ there exists a subgroup $G \subseteq \mathbb{R}$ such that $|\mathbb{R} / G|=\kappa$.

Proof. Let $H \subseteq \mathbb{R}$ be a Hamel base (i.e. a base of $\mathbb{R}$ as a linear space over $\mathbb{Q}$ ). Take $Z \in[H]^{\kappa}$ such that $|H \backslash Z|=\mathfrak{c}$ and let $G$ be the linear subspace of $\mathbb{R}$ generated by $H \backslash Z$. Then $\mathbb{R} / G$ is isomorphic to the space generated by $Z$, in particular it has cardinality $\kappa$.

Lemma 2.9. There exists a subgroup $B \subseteq \mathbb{R}$ such that

- $\mathbb{R} / B \simeq \mathbb{R}$

- $B$ is a Bernstein set in $\mathbb{R}$.

Proof. The approach is similar to the proof of Lemma 2.8. By straightforward transfinite induction we construct Bernstein sets $B_{0}$ and $B_{1}$ such that $B_{0} \cup B_{1}$ is linearly independent over $\mathbb{Q}$. Let $B$ be the linear space generated by $B_{0}$. As $\left|B_{1}\right|=\mathfrak{c}$, the quotient $\mathbb{R} / B$ is isomorphic to the linear space over $\mathbb{Q}$ generated by $\mathfrak{c}$ many independent vectors (more precisely: $B_{0} \cup B_{1}$ can be expanded to a Hamel base $H$, the generators are vectors from $H \backslash B_{0}$ and this set contains $\left.B_{1}\right)$. But $\mathbb{R}$ is also a linear space generated by $\mathfrak{c}$ many independent vectors over $\mathbb{Q}$ and every two such spaces are isomorphic.

We have to show that $B$ is a Bernstein set. Clearly, $B$ intersects all perfect sets, because $B_{0} \subseteq B$ does. On the other hand, if $B$ contained a perfect set $P$, then each non-zero coset of $B$, say $z+B$ for $z \notin B$, would contain $z+P$. But then $z+P$ would be a perfect set disjoint with $B$.

Remark 2.10. Let $B$ be a group which is a Bernstein set and let $C$ be a proper nonempty subset of $\mathbb{R} / B$. Then $\pi^{-1}[C]$ is a Bernstein set in $\mathbb{R}$. This is because 
$B$ is Bernstein, so all cosets of $B$ are Bernstein sets. Therefore $\pi^{-1}[C]$ intersects all perfect sets (because, as $C \neq \emptyset$, it contains a coset) but does not contain any perfect set (because, as $C \neq \mathbb{R} / B$, there is a coset disjoint with $\pi^{-1}[C]$ ).

We are going to show that, considering questions similar to Theorem 2.3 and being able to construct a partition into coverings, we can at no cost assume that all pieces are Bernstein sets. The simplest and special case of our theorem is that the existence of a partition of $\mathbb{R}$ into $\kappa$ many $\lambda$-coverings implies the existence of a partition of $\mathbb{R}$ into $\kappa$ many $\lambda$-coverings which are Bernstein sets. We want, however, a more general statement. First of all, we allow different $\lambda$ 's for different pieces of our partitions. Apart from that, we also want our theorem not only to preserve the fact of being a $\lambda$-covering, but also want our Bernstein sets to be coverings for exactly the same $\lambda$ 's, as corresponding sets in the original partition. We shall occasionally use that to construct partitions into sets which have a very weak covering condition.

Observe that for any set $A \subsetneq \mathbb{R}$ we may define a cardinal

$$
\min \{\lambda \leq \mathfrak{c}: A \text { is not a } \lambda \text {-covering }\}
$$

which precisely characterizes for which $\lambda \leq \mathfrak{c} A$ is a $\lambda$-covering. This is a meaningful definition for any proper subset of $\mathbb{R}$ (because $\mathbb{R}$ is the only $\mathfrak{c}$-covering).

Theorem 2.11. For every partition $\left\{A_{\alpha}: \alpha<\kappa\right\}(\kappa>1)$ of $\mathbb{R}$ into nonempty sets there exists a partition $\left\{B_{\alpha}: \alpha<\kappa\right\}$ of $\mathbb{R}$ into Bernstein sets such that

$$
\min \left\{\lambda: A_{\alpha} \text { is not a } \lambda \text {-covering }\right\}=\min \left\{\lambda: B_{\alpha} \text { is not a } \lambda \text {-covering }\right\} \text {. }
$$

Proof. Let $B$ be a Bernstein group as in Lemma 2.9. As $\mathbb{R} / B$ and $\mathbb{R}$ are group isomorphic, we may assume that $\left\{A_{\alpha}: \alpha<\kappa\right\}$ is partition of $\mathbb{R} / B$. Let $B_{\alpha}=\pi^{-1}\left[A_{\alpha}\right]$, where $\pi: \mathbb{R} \rightarrow \mathbb{R} / B$ is the quotient epimorphism. By Proposition 2.7, we obtain that $\min \left\{\lambda: A_{\alpha}\right.$ is not a $\lambda$-covering $\}=\min \left\{\lambda: B_{\alpha}\right.$ is not a $\lambda$-covering $\}$.

The fact that each $B_{\alpha}$ is a Bernstein set follows from Remark 2.10 and from the fact that $A_{\alpha}$ is nonempty and proper subset of $\mathbb{R}$.

As an immediate corollary we obtain something which requires a technical argument in [4].

Corollary 2.12 (Kraszewski-Rałowski-Szczepaniak-Żeberski, [4]). There exists a partition of $\mathbb{R}$ into Bernstein sets $A, B$ such that none of them is a 2-covering.

Proof. Consider a partition of $\mathbb{R}$ into two pieces $R_{0}=\bigcup_{k \in \mathbb{Z}}[2 k, 2 k+1), R_{1}=$ $\bigcup_{k \in \mathbb{Z}}[2 k-1,2 k)$ which are not 2 -coverings, and apply the theorem.

The following theorem in particular answers Question 2.4.

Theorem 2.13. For every cardinal $\kappa$ such that $\omega \leq \kappa \leq \mathfrak{c}$, there exists a Bernstein set which is a< $a$-covering and is not a $\kappa$-covering. In particular, a Bernstein $\omega_{1}$-covering exists, if and only if, $\mathfrak{c}>\omega_{1}$.

Proof. By Theorem 2.11 it is enough to find any set in $\mathbb{R}$ which is a $<\kappa$-covering and is not a $\kappa$-covering. For $\kappa=\mathfrak{c}$ (which answers Question 2.4), simply take $\mathbb{R} \backslash\{0\}$.

For arbitrary infinite $\kappa<\mathfrak{c}$, we use Lemma 2.8 to construct a group $G \subseteq \mathbb{R}$ such that $|\mathbb{R} / G|=\kappa$. Then a complement of a singleton in $\mathbb{R} / G$ is a a $<\kappa$-covering (because every proper subset of $\mathbb{R} / G$ can be translated into it) and is not a $\kappa$-covering 
(because the only $\kappa$ covering is the whole group $\mathbb{R} / G$ ). Then, by taking the preimage and using Proposition 2.7, we transfer this example from the quotient to $\mathbb{R}$.

The previous theorem provides us just one Bernstein covering, while the authors of [4] were interested in constructing partitions into such coverings. If $\mathfrak{c}$ is regular, Theorem 2.3 is in a sense the best possible - we have as many sets in the partition as possible and their covering condition is as strong as possible. However, if $\mathfrak{c}=\omega_{\omega_{1}}$, this theorem gives us only a partition into $\omega$-coverings. On the other hand, we know that (Bernstein) $<\mathfrak{c}$-coverings exist in ZFC, but if $\mathfrak{c}$ is singular, by Proposition 2.6 no partitions into such coverings are possible. Still we can construct partitions into $\kappa$-coverings for a fixed $\kappa<\mathfrak{c}$ in ZFC, but at the cost of reducing the number of sets in the partitions, as compared to Theorem 2.3.

Theorem 2.14. For every infinite $\kappa<\mathfrak{c}$ there exists a partition of $\mathbb{R}$ into $\kappa^{+}$many $\kappa$-coverings which are Bernstein sets.

Proof. By Theorem 2.11 it is enough to construct a partition of $\mathbb{R}$ into $\kappa^{+}$many $\kappa$-coverings. As $\kappa^{+} \leq \mathfrak{c}$, by Lemma 2.8 we can to find a subgroup $G \subseteq \mathbb{R}$ with $|\mathbb{R} / G|=\kappa^{+}$. Applying Lemma 2.5 in $\mathbb{R} / G$, we can find a partition of $\mathbb{R} / G$ into $\kappa^{+}$ many $\kappa$-coverings and this partition can be easily transferred to $\mathbb{R}$ by Proposition 2.7 .

\section{Bernstein sets AND other Algebraic PRoperties}

3.1. Earlier results. In [8] the authors construct subsets $\mathbb{R}$ which are completely nonmeasurable with respect to Lebesgue measure and Baire property and have some special additive properties. Following the terminology from [8], a set $X \subseteq \mathbb{R}$ is completely nonmeasurable with respect to a $\sigma$-ideal $\mathcal{I}$, if neither $X$, nor its complement contain a Borel set which is not in $\mathcal{I}$. In the case of Lebesgue measurability (or, more precisely, of the ideal of Lebesgue measure zero sets) this is equivalent to saying that $X$ has inner measure zero and outer full measure.

This section is devoted to constructing Bernstein sets having the same or similar additive properties as considered in [8]. It is easy to check (see similar arguments in [5]) that every Bernstein set is completely nonmeasurable with respect to every $\sigma$-ideal $\mathcal{I}$ with a base consisting of $\boldsymbol{\Pi}_{1}^{1}$ sets. In particular, it is completely nonmeasurable with respect to Lebesgue measure and Baire category. Therefore, our results are improvements of those obtained in [8]. Occasionally, we will also improve the number of sets in considered partitions - sometimes we are able to construct partitions into $\mathfrak{c}$ many Bernstein sets with desired property, while the methods used in [8] can only produce countable partitions.

It should be noted that the results from [8] utilize very elegant geometric arguments. The general method used there is to construct in $\mathbb{R}^{2}$ a set or a partition into sets having certain algebraic properties in a definable way and then apply a theorem of Cichon and Szczepaniak from [3]. This theorem states that the image via linear isomorphism (over $\mathbb{Q}$ ) from $\mathbb{R}^{2}$ to $\mathbb{R}$ of a set $X \subseteq \mathbb{R}^{2}$ is completely nonmeasurable, if only interior of $X$ and the interior of $\mathbb{R}^{2} \backslash X$ are nonempty. Taking such images preserves simple algebraic properties, thus produces completely nonmeasurable sets with desired algebraic properties.

Our approach is similar and based on constructions of Rałowski, Szczepaniak and Żeberski - we will use the same or similar definable sets, but instead of the theorem of Cichoń and Szczepaniak, we will apply Lemma 2.9 and Remark 2.10. This will 
work the same way, i.e. will preserve algebraic properties but will produce Bernstein sets instead of merely completely nonmeasurable ones. We also have more liberty with constructing definable examples - the theorem of Cichon and Szczepaniak can be applied to sets such that the interiors of the set and of the complement are nonempty. Application of our method requires only the set and the complement themselves to be nonempty (but possibly nowhere dense).

3.2. The method. We shall say that a property of a subset of a group (or a family or a sequence of sets) is algebraic if it is preserved at taking preimages of sets via (quotient) epimorphisms. In this terminology, Proposition 2.7 says that being a $\kappa$-covering is an algebraic property. Other examples of algebraic properties are:

- $A+A=A$,

- $A+B$ is the whole group,

- disjointness of a family of sets,

- being a strictly increasing sequence of sets.

We will now outline our general method. We intentionally avoid formulating it as a precisely stated theorem and put it in a little bit vague way. This is done because a formal definition of algebraic property would require introducing some second-order logic, which we want to avoid here. Our general approach looks as follows.

(1) We construct a set, a family or a sequence of sets in $\mathbb{R}$ or $\mathbb{R}^{2}$ (remember that all $\mathbb{R}^{n}$ 's are group isomorphic) with some algebraic property. This will be done in a definable way and our examples will be nonempty sets with nonempty complements.

(2) We take a group $B$ as in Lemma 2.9 along with the quotient epimorphism $\pi: \mathbb{R} \rightarrow \mathbb{R} / B$ and find an analogous object (i.e. a set, a family or a sequence) with the same property in the quotient $\mathbb{R} / B$.

(3) Taking preimages via $\pi$ of sets constructed in (1) will preserve our algebraic property but, by Remark 2.10, the preimages of all sets in question will be Bernstein sets.

It should be clear that the method does what it is intended to do. As we remarked earlier, being a $\kappa$-covering is an algebraic property, thus a particular instance of application of this method is for example Theorem 2.11 along with its proof. In the sequel we will only outline the proofs which fits to this scheme (that is, we will indicate what definable sets we take and what is the algebraic property in question) than formulate it precisely.

3.3. Applications. To illustrate our method we present a list of its possible applications. The following list of propositions is meant to be rather a set of examples than a complete list of applications that the author can think of.

Proposition 3.1. There exists a Bernstein set such that $B+B=B$ and $B-B=\mathbb{R}$.

Proof. We have to construct a Bernstein set with an algebraic property. Observe that $[0,+\infty)$ has the same property in $\mathbb{R}$.

Proposition 3.2. There exists a partition $\left\{B_{\xi}: \xi<\mathfrak{c}\right\}$ of $\mathbb{R}$ into Bernstein sets such that $B_{\xi}+B_{\xi}=B_{\xi}$ for $\xi<\mathfrak{c}$. 
Proof. Each half-line in $\mathbb{R}^{2}$ starting in $\langle 0,0\rangle$ (and containing the endpoint or not) has the algebraic property we are interested in ([8]). Therefore we may partition $\mathbb{R}^{2}$ into such half-lines without the endpoint and add $\langle 0,0\rangle$ to one of them.

Proposition 3.3. There exists a partition $\left\{B_{n}: n \in \omega\right\}$ of $\mathbb{R}$ into Bernstein sets such that $B_{n}+B_{n}=B_{n}$ for $n \in \omega$.

Proof. Similar to the previous one - the angle between two half-lines (containing $\langle 0,0\rangle$ or not) has the same algebraic property ([8]). Therefore we may partition $\mathbb{R}^{2}$ into such "open" angles, the "open" half-lines forming their boundaries and toss $\langle 0,0\rangle$ into one of them.

Proposition 3.4. There exists a partition $\left\{B_{\xi}: \xi<\mathfrak{c}\right\}$ of $\mathbb{R}$ into Bernstein sets such that for every $\xi<\mathfrak{c}, B_{\xi}+B_{\xi}=\mathbb{R}$.

Proof. Let $S_{r}=\left\{\langle x, y\rangle \in \mathbb{R}^{2}: x^{2}+y^{2}=r^{2}\right\}$ and $C_{r}=\left\{\langle x, y\rangle \in \mathbb{R}^{2}: x^{2}+y^{2} \leq r^{2}\right\}$, then $S_{r}+S_{r}=C_{2 r}$. Therefore, for every $r \in[0,1)$, the set

$$
X_{r}=\bigcup_{n \in \mathbb{N}} S_{r+n}
$$

has the property that $X_{r}+X_{r}=\mathbb{R}^{2}$, so we have constructed a partition of $\mathbb{R}^{2}$ into $\mathfrak{c}$ many sets with desired algebraic property.

Proposition 3.5. There exists a set $B \subseteq \mathbb{R}$ such that all sets of the form

$$
B^{(n)}=B+B+\ldots+B
$$

(where the sum is of length n) form an increasing sequence of Bernstein sets and $\bigcup_{n \in \omega} B^{(n)}=\mathbb{R}$.

Proof. The set $(-\infty, 1]$ has the desired algebraic property in $\mathbb{R}$.

\section{REFERENCES}

1. T. J. Carlson, Strong measure zero and strongly meager sets, Proceedings of the American Mathematical Society 118 (1993), no. 2, 577-586.

2. J. Cichoń, A. Jasiński, A. Kamburelis, and P. Szczepaniak, On translations of subsets of the real line, Fundamenta Mathematicae 130 (2002), no. 6, 1833-1842.

3. J. Cichon and P. Szczepaniak, When is the unit ball nonmeasurable?, Preprint.

4. J. Kraszewski, R. Rałowski, P. Szczepaniak, and Sz. Żeberski, Bernstein sets and $\kappa$-coverings, Preprint, http://www.math.uni.wroc.pl/〜kraszew/sources/papers/BernsteinCov6.pdf.

5. M. Kysiak, Nonmeasurable algebraic sums of sets of reals, Colloquium Mathematicum 102 (2005), no. 1, 113-122.

6. K. Muthuvel, Application of covering sets, Colloquium Mathematicum 80 (1999), 115-122.

7. A. Nowik, Some topological characterizations of omega-covering sets, Czechoslovak Mathematical Journal 50 (2000), no. 125, 865-877.

8. R. Rałowski, P. Szczepaniak, and Sz. Żeberski, A generalization of Steinhaus theorem and some nonmeasurable sets, Preprint.

Institute of Mathematics, University of WarsaW, Ul. Banacha 2, 02-097 Warszawa, POLAND

E-mail address: mkysiak@mimuw.edu.pl 